\title{
CARACTERÍSTICAS MORFOLÓGICO-VEGETATIVAS DE CULTIVARES DE COQUEIRO ${ }^{1}$
}

\author{
AMANDA DA SILVA ALVES ${ }^{2}$, WILSON MENEZES ARAGÃO ${ }^{3}$, \\ CARINA MENDES LOIOLA ${ }^{4}$, GABRIELE TORINO PEDROSO ${ }^{5}$
}

RESUMO - O presente estudo foi realizado a partir de agosto de 1997 até dezembro de 2000, na região do Platô de Neópolis, Sergipe, com a finalidade de avaliar o comportamento de cultivares de coqueiro (Cocos nucifera L.) em diferentes anos agrícolas para as características: número de folhas vivas (NFV), número de folhas emitidas (NFE), circunferência do coleto (CC), comprimento da folha 3 (CF3), comprimento do limbo da folha 3(CLF3), comprimento do pecíolo da folha 3 (CPF3), número de folíolos da folha 3 (NFoF3) e comprimento do folíolo da folha 3 (CFoF3). Utilizou-se o delineamento em parcelas subdivididas, dispostas em blocos ao acaso, com quatorze repetições, quatorze tratamentos e três anos de avaliação, utilizando-se de 16 plantas úteis por parcela. Os resultados das análises demonstraram que, para todas as características, ocorreram diferenças significativas a $1 \%$ entre as cultivares e anos, e que a interação cultivar $\mathrm{x}$ ano foi significativa a $5 \%$ para NFE, e significativa a $1 \%$ para as demais características, indicando um comportamento diferente das cultivares em relação aos anos.

Termos de indexação: variedades, híbridos, melhoramento genético.

\section{MORPHOLOGIC-VEGETATIVE CHARACTERISTICS OF COCONUT CULTIVARS}

ABSTRACT -The present work was carried out from August 1997 to December 2000, at the "Plato of Neópolis", State of Sergipe, aiming to evaluate the characteristics of number of alive leaves (NFV), number of born leaves (NFE), stem diameter (CC), length of leaf 3 (CF3) length of the leaf blade of leaf 3 (CLF3), length of the petiole of leaf 3 (CPF3), and number of leaflets of leaf 3 (CfoF3) of coconut (Cocus nucifera L.) cultivars, in different agricultural years. A split plot randomized block design with four replications, fourteen treatments and three years of evaluations was used. Records were taken in 16 plants/plot. Statistic analysis of data showed significant differences among cultivars and years for all the evaluated characteristics. Highly significant interaction between cultivar $\mathrm{x}$ year was found for NFE and significant interactions for the remained characteristics, pointing out for a diverse behavior of cultivars in relation to the year crop.

Index terms: variety, hybrid, breeding.

\section{INTRODUÇ̃̃O}

A cultura do coqueiro é cultivada em 86 países, sendo típica de clima tropical (Camboim, 2003). A origem da espécie é passível de discussão. Segundo Purseglove (1972), o sudeste Asiático é tido como a região de origem do coqueiro, principalmente as ilhas entre os Oceanos Índico e Pacífico, embora outros autores coloquem o nordeste da América do Sul também como centro de origem.

Assim, como as demais palmáceas, o coqueiro encontra condições favoráveis ao seu desenvolvimento entre as latitudes $20^{\circ} \mathrm{N} \mathrm{e} 20^{\circ} \mathrm{S}$ (Passos, 1998), com temperatura média anual em torno de $27^{\circ} \mathrm{C}$ (Child, 1974) e umidade relativa superior a $60 \%$. É uma cultura bastante exigente em luz, em torno de 2.000 horas anuais (Passos \& Silva, 1991) e desenvolve-se bem em locais onde a precipitação anual está na faixa de 1.700 a $2.200 \mathrm{~mm}$, com o mínimo de $130 \mathrm{~mm} / \mathrm{mês}$; menos que isso, faz-se necessário uso de irrigação (Frémond et al., 1975).

Sob condições ambientais desfavoráveis, a fisiologia e morfologia do coqueiro podem ser afetadas, podendo, em algumas situações, reduzir o número de folhas (Frémond et al., 1975) ou até mesmo alterar o diâmetro do caule (Ferri, 1973). Ainda, segundo Passos (1998), em condições de estiagem prolongada, ocorre diminuição no fenorritmo foliar e redução da longevidade da folha.

A alteração no desempenho relativo dos genótipos, em virtude de diferenças entre ambientes, denomina-se interação genótipo x ambiente. Segundo Vencovsky \& Barriga (1998) e Cruz \& Regazzi (1994), quando não existe essa interação, significa dizer que a cultivar se adapta bem aos diferentes ambientes de cultivo. Quando ocorre interação, significa que as melhores cultivares num determinado local não o sejam em outros.

O melhoramento tem o objetivo de desenvolver cultivares mais produtivas, adaptadas às condições ambientais diversas $\mathrm{e}$ a sistemas de produção com baixo emprego de tecnologias (World Bank, 1991). Por isso, é de fundamental importância conduzir

\footnotetext{
Trabalho (076-06). Recebido em 05-06-2006. Aceito para publicação em : 24-05-2007.

2 Engenheira Agrônoma graduada pela Universidade Federal de Sergipe, Rua das Papoulas, nº 94, Conjunto Beira Rio, Bairro Inácio Barbosa.Aracaju-SE. CEP: 49040450 amanda_ama@uol.com.br

${ }^{3}$ Engenheiro Agrônomo Pesquisador da Embrapa Tabuleiros Costeiros, Av. Beira Mar 3250. Caixa postal 44, 49001-970, Aracaju, Sergipe, Brasil. Aracaju-SE aragaowm@.cpatc.embrapa.br

${ }^{4}$ Engenheira Agrônoma, Bolsista CNPq, Av. Beira Mar 3250. Caixa postal 44, 49001-970, Aracaju, Sergipe, Brasil. Aracaju-SE carina_loiola@yahoo.com.br

${ }_{5}^{5}$ Estudante de Engenharia Agronômica da Universidade Federal de Sergipe bolsista do CNPq, Av. Beira Mar 3250. Caixa postal 44, 49001-970,

Aracaju, Sergipe, Brasil. Aracaju-SE gabrieletp@yahoo.com.br
} 
experimentos multilocais, dentro e entre regiões avaliadas em diferentes anos agrícolas, para se selecionarem as variedades mais produtivas e de maior estabilidade em diferentes ambientes.

Este trabalho objetivou avaliar o comportamento de cultivares de coqueiro em diferentes anos agrícolas para características morfológico-vegetativas.

\section{MATERIAL E MÉTODOS}

O ensaio foi implantado em agosto de 1997 e é conduzido até os dias de hoje, sendo que, para o presente trabalho, foi avaliado o período de março de 1997 a dezembro de 2000, na Agreste Ltda., localizada no Platô de Neópolis-SE, em parceria com a Embrapa Tabuleiros Costeiros, utilizando-se das seguintes cultivares: Anão-Amarelo do Brasil de Gramame (AABrG), AnãoAmarelo da Malásia (AAM), Anão-Vermelho de Camarões (AVC), Anão- Vermelho do Brasil de Gramame (AVBrG), Anão-Vermelho da Malásia (AVM), Anão- Verde do Brasil de Jiqui (AVeBrJ), AABrG x Gigante do Brasil da Praia do Forte (GBrPF), AABrG x Gigante do Oeste Africano (GOA), AABrG x Gigante da Polinésia (GPY), AVBrG x GBrPF, AVBrG x GOA, AVBrG x GPY, AVBrG x Gigante de Rennell (GRL) e AVeBrJ x GBrPF.

O clima da região é do tipo A's, com uma precipitação média anual de $1.270,3 \mathrm{~mm}$, dos quais $71,8 \%$ e $28,2 \%$ ocorrem nos períodos de chuva (abril a setembro) e seca (outubro a março), respectivamente. A evapotranspiração média anual é de 177,09 $\mathrm{mm}$, com temperaturas médias das máximas de $30^{\circ} \mathrm{C}$ e média das mínimas de $19,4^{\circ} \mathrm{C}$, sendo a média anual em torno de $24,7^{\circ} \mathrm{C}$. A umidade relativa média é de $76,67 \%$.

O solo é do tipo ARGISSOLO Vermelho-Amarelo com baixa fertilidade natural, apresentando, respectivamente, nas camadas de 0-10 cm e 10-20 cm, as seguintes composições químicas: pH em água - 6,20 e 5,69; P - 11,9 e 5,6 mg.dm ${ }^{-3} ; \mathrm{K}$ 53,17 e 30,62 mmol $_{\text {сKg }}{ }^{-1} ; \mathrm{Ca}-14,5$ e 9,0 mmol $_{\text {сKg }}^{-1} ; \mathrm{Mg}-6,2$ e 4,7 $\mathrm{mmol}_{\mathrm{cKg}^{-1}}{ }^{-1} \mathrm{Al}-0,6$ e 1,2 mmol $_{\mathrm{cKg}}{ }^{-1}$ e M.O. $-1,5$ e 9,5 g. $\mathrm{Kg}^{-1}$

O delineamento experimental foi em parcelas subdivididas, dispostas em blocos ao acaso, com quatro repetições e quatorze tratamentos, e três anos de avaliação, utilizando 16 plantas úteis por parcela. Implantou-se uma bordadura em torno das repetições com as cultivares correspondentes à das parcelas úteis. $\mathrm{O}$ espaçamento de plantio foi de $7,5 \mathrm{~m}$ para os anões e $8,5 \mathrm{~m}$ para híbridos, dispostos em triângulo eqüilátero.

O controle de plantas invasoras consistiu na aplicação de glifosate na coroa da planta e roçagem mecânica nas entrelinhas de plantio. A adubação foi realizada mensalmente, com base na análise foliar (Sobral, 1998), e a irrigação consistiu na aplicação de 150 litros de água/dia/planta, no período seco do ano.

As avaliações foram realizadas a cada três meses, sendo que a primeira só foi realizada seis meses após a implantação do experimento, totalizando assim quatro avaliações por ano, durante três anos, até o primeiro florescimento das cultivares que, segundo Aragão et al. (2002), ocorre para os anões e os híbridos, em média, aos 2,6 anos e 3,1 anos, respectivamente. Os caracteres de avaliação foram: número de folhas vivas (NFV), número de folhas emitidas (NFE), circunferência do coleto (CC), comprimento da folha 3 (CF3), comprimento do limbo da folha 3(CLF3), comprimento do pecíolo da folha 3 (CPF3), número de folíolos da folha 3 (NFoF3) e comprimento do folíolo da folha 3 (CFoF3).

$\mathrm{O}$ NFV corresponde às folhas $50 \%$ ou mais abertas até $50 \%$ ou mais vivas; o NFE corresponde às folhas emitidas a partir da última avaliação até a folha de número um que está 50\% ou mais aberta; CC é a medida no coleto das mudas ou do caule em plantas adultas a $5 \mathrm{~cm}$ do solo, com auxílio de uma fita métrica; o CF3 é a medida em metros do ponto de inserção do pecíolo no caule até o ponto de inserção do último par de folíolos; o CLF3 é medida em metros da área que vai do ponto de inserção do primeiro par de folíolos até o último par; o CPF3 é a medida em metros do ponto de inserção do pecíolo no caule até o primeiro par de folíolos; o CFoF3 é a medição, em centímetros, de três folíolos localizados na região central da folha; o NFoF3 é a contagem do número de folíolos da folha. A utilização da folha três deve-se por ser a folha de maior atividade metabólica nessa fase de desenvolvimento.

Com base nas médias das cultivares, foi realizada a análise de variância por característica e ano, verificando a relação dos quadrados médios residuais entre os anos para a realização da análise conjunta. Para que essa análise ocorra, essa relação tem de ser obrigatoriamente igual ou menor que sete (Gomes, 1985). Após análise de variância conjunta, as médias das cultivares e dos anos foram comparadas pelo teste de Tukey, a 5\% de probabilidade (Gomes, 1985).

A partir da análise de variância das diversas características, determinou-se o coeficiente de determinação genotípica (bp) (Falconer, 1987).

\section{RESULTADOS E DISCUSSÃO}

De acordo com o resumo da análise de variância (Tabela1), observa-se que, para todas as características, ocorreram diferenças significativas a $1 \%$ entre as cultivares e entre os anos, pelo teste $\mathrm{F}$. A interação cultivar $\mathrm{x}$ ano foi significativa a $5 \%$ para NFE e a $1 \%$ para as demais características, indicando um comportamento diferente das cultivares em relação aos anos.

Observa-se ainda, na Tabela 1, que os coeficientes de variação $(\mathrm{CV} \%)$, em geral, foram baixos para as diferentes características, indicando uma boa precisão experimental.

O coeficiente de determinação genotípica (bp), em geral, foi alto para as diversas características, exceto para $\operatorname{NFE}(b p=0,3)$, o que implica maior dificuldade nos trabalhos de seleção de cultivares para essa característica (Tabela1).

$\mathrm{O}$ aumento do NFV foi constante entre os anos (Tabela 2), para a CC (Tabela 3), para o CF3 e CPF3 (Tabela 4), e para o CFoF3 e NFoF3 (Tabela 5) ocorreu aumento entre todos os anos, sendo que o maior aumento foi do ano 2 para o ano 3 , para o NFE (Tabela 2), o maior aumento ocorreu do ano 3 para o ano 2 , e para o CLF3 (Tabela 4) o maior aumento ocorreu do ano 2 para 0 ano 1.

Os híbridos tenderam a apresentar maior NFV em relação aos anões, tanto no ano 1 como no ano 2, enquanto no ano 3 os híbridos apresentam comportamento intermediário, apesar de não ter ocorrido diferença significativa, pelo teste de Tukey, $\mathrm{p} \leq 0,05$ entre a maioria das cultivares. No ano 2 , os híbridos de anão- 
amarelo tenderam a apresentar maior NFV (em média 16,1) em relação aos híbridos de anão-vermelho (em média 15,3). Esse comportamento difere do observado no trabalho de Ramos et al. (2004), segundo o qual os híbridos dos mesmos anões só apresentaram, em média, 8 e 9 folhas vivas até o segundo ano, respectivamente. No ano três, os anões-amarelo e verde e os respectivos híbridos, tenderam a apresentar maior NFV em relação aos anões-vermelhos e aos híbridos desses anões (Tabela 2).

Para a característica NFE, as cultivares não diferiram entre si, pelo teste de Tukey, $\mathrm{p} \leq 0,05$, nos anos 1 e 2 (em média 12 folhas), mas, no ano 3, observa-se que o AABrGxGOA, AAM, AABrG X GBrPF, AVeBrJ x GBrPF, AVC e o AVM tenderam a apresentar maiores NFE (Tabela 2).

O NFE médio, independentemente do ano, está de acordo com os resultados apresentados por Passos \& Silva (1991), segundo os quais o coqueiro emite de 12 a 15 folhas por ano, embora, ainda segundo Passos (1998), o coqueiro-anão pode emitir 18 folhas por ano.

Para a CC, observa-se que, entre os híbridos, praticamente não houve diferença significativa, à exceção dos anos $2 \mathrm{e} 3$, em que o AABrGxGOA diferiu significativamente dos demais híbridos. Entre os anões, nos anos 1 e 2, o AVC diferiu significativamente dos demais anões, apresentando as mesmas circunferências (Tabela 3 ).

Os híbridos apresentaram maior CF3, não diferindo entre si, mas, sendo significativamente superior aos anões, independentemente do ano. No terceiro ano, o comprimento da folha dos híbridos, quando comparado com os anões, variou de 4,1 a 4,5 m e de 3,0 a 3,6 m, respectivamente. Entre os anões, o $\mathrm{AVC}$ apresentou, em números reais, menor $\mathrm{CF} 3$, independentemente do ano, sendo que, no terceiro ano, só não diferiu significativamente do AAM, pelo teste de Tukey, a $\mathrm{p} \leq 0,05$
(Tabela 3).

Observa-se que os híbridos apresentaram um maior CLF3, principalmente os híbridos de anão-vermelho. Entre os anões, o AVBrG tendeu a apresentar maior CLF3, e o AVC, o menor comprimento, nos três anos (Tabela 4) .

Para a característica CPF3, observa-se que o maior aumento ocorreu do ano 3 em relação ao ano $2(120,1 \%)$, comportamento diferente do observado para as demais características.

Em geral, os híbridos e os anões apresentaram maiores e menores CPF3, respectivamente (Tabela 4). Apesar de que os híbridos AVeBrJ x GBrPF, AVBrG x GOA, AABrG x GPY,AABrG x GOA, AABrG x GBrPF não se diferenciaram $(p \leq 0,05)$ do anãoverde, e os dois últimos híbridos, do AVM. A média do CPF3 das cultivares foi de $1,1 \mathrm{~m}$ com amplitude de 1,4 a $0,7 \mathrm{~m}$.

Não houve diferença significativa entre as cultivares para o NFoF3, no ano 1. Já no ano $2(152,5)$ e no ano $3(185,7)$, o híbrido AVBrG x GBrPF apresnetou o maior NFoF3, apesar de não diferir, $\mathrm{p} \leq 0,05$, dos demais híbridos e do AVBrG. O AVC, no ano $2(101,4)$ e no ano $3(117,6)$, e o AVeBrJ no ano $3(136,7)$ apresentaram os menores NFoF3. Apesar de não diferirem da maioria dos anões.

Observa-se, na Tabela 5, que os híbridos tenderam a apresentar maior CFoF3 quando comparados com os anões, principalmente os híbridos de anões-vermelhos. Entre os anões, o $\mathrm{AVeBrJ}$ e o AVC apresentaram maior e menor $\mathrm{CFoF} 3$, respectivamente (Tabela 5).

TABELA 1 - Resumo da análise de variância para número de folhas vivas (NFV), número de folhas emitidas (NFE), circunferência do coleto (CC), comprimento da folha 3 (CF3), número de folíolos da folha 3 (NFoF3), comprimento dos folíolos da folha 3 (CFoF3), comprimento do limbo da folha 3 (CLF3), comprimento do pecíolo da folha 3 (CPF3), no período de 1997 a 2000, em Neópolis-SE.

\begin{tabular}{|c|c|c|c|c|c|c|c|c|c|}
\hline \multirow[t]{2}{*}{$\mathrm{FV}$} & \multirow[t]{2}{*}{ GL } & \multicolumn{7}{|c|}{$\mathrm{QM}$} & \multirow[b]{2}{*}{ CPF3 } \\
\hline & & NFV & NFE & $\mathrm{CC}$ & CF3 & NFoF3 & CFoF3 & CLF3 & \\
\hline Cultivar (C) & 13 & $9,5 * *$ & $0,1^{* *}$ & $2666,5^{* *}$ & $2,50 * *$ & $2344,8^{* *}$ & $1618,4^{* *}$ & $1,63 * *$ & 0,19 \\
\hline Erro 1 & 39 & 0,4 & 0,1 & 72,9 & 0,03 & 90,6 & 23,7 & 0,03 & 0,01 \\
\hline Ano (A) & 2 & $2359,9 * *$ & $2,4 * *$ & $14702,8 * *$ & $93,50 * *$ & $131476,9^{* *}$ & $10854,2 * *$ & $55,60 * *$ & 10,70 \\
\hline Interação CxA & 26 & $1,2 * *$ & $0,2^{*}$ & $328,2^{* *}$ & $0,06^{* *}$ & $338,4 * *$ & $42,7^{* *}$ & $0,09 * *$ & 0,06 \\
\hline Erro 2 & 84 & 0,3 & 0,1 & 36,8 & 0,03 & 125,7 & 15,9 & 0,03 & 0,02 \\
\hline CV 1 & & 4,60 & 11,10 & 9,30 & 6,10 & 7,80 & 5,60 & 8,40 & 15,30 \\
\hline CV 2 & & 3,70 & 12,20 & 6,60 & 5,70 & 9,20 & 4,50 & 7,50 & \\
\hline bp & & 0,90 & 0,30 & 0,90 & 1,00 & 0,90 & 0,90 & 0,97 & \\
\hline
\end{tabular}

* Significativo ao nível de $5 \%$ de probabilidade pelo teste $\mathrm{F}$

** Significativo ao nível de $1 \%$ de probabilidade pelo teste 
TABELA 2 - Dados médios do número de folhas vivas e número de folhas emitidas em cultivares de coqueiro, no período de 1997 a 2000, em Neópolis-SE.

\begin{tabular}{lllllll}
\hline \multirow{2}{*}{ Cultivar } & \multicolumn{9}{c}{ NFV } & \multicolumn{2}{c}{ NFE } \\
\cline { 2 - 7 } & \multicolumn{1}{c}{ Ano 1} & \multicolumn{1}{c}{ Ano 2} & \multicolumn{1}{c}{ Ano 3} & $12,0 \mathrm{a}$ & $12,0 \mathrm{a}$ & $14,8 \mathrm{ab}$ \\
\hline AABrG & $8,0 \mathrm{abc}$ & $15,0 \mathrm{ab}$ & $22,0 \mathrm{ab}$ & $12,0 \mathrm{a}$ & $12,0 \mathrm{a}$ & $16,0 \mathrm{a}$ \\
AAM & $8,0 \mathrm{ab}$ & $14,2 \mathrm{bc}$ & $22,2 \mathrm{a}$ & $14,0 \mathrm{a}$ & $12,0 \mathrm{a}$ & $16,0 \mathrm{a}$ \\
AVC & $6,2 \mathrm{~d}$ & $12,2 \mathrm{~d}$ & $18,7 \mathrm{e}$ & $12,0 \mathrm{a}$ & $12,0 \mathrm{a}$ & $12,0 \mathrm{~b}$ \\
AVBrG & $7,5 \mathrm{bcd}$ & $14,0 \mathrm{bc}$ & $20,0 \mathrm{de}$ & $12,0 \mathrm{a}$ & $12,0 \mathrm{a}$ & $12,0 \mathrm{~b}$ \\
AVM & $7,0 \mathrm{~cd}$ & $13,2 \mathrm{~cd}$ & $20,5 \mathrm{~cd}$ & $12,8 \mathrm{a}$ & $12,0 \mathrm{a}$ & $14,0 \mathrm{ab}$ \\
AVeBrJ & $7,2 \mathrm{bcd}$ & $14,2 \mathrm{bc}$ & $22,0 \mathrm{ab}$ & $14,0 \mathrm{a}$ & $12,0 \mathrm{a}$ & $16,0 \mathrm{a}$ \\
AABrGxGBrPF & $8,2 \mathrm{abc}$ & $16,0 \mathrm{a}$ & $21,5 \mathrm{abc}$ & $12,0 \mathrm{a}$ & $12,0 \mathrm{a}$ & $16,0 \mathrm{a}$ \\
AABrGxGOA & $8,5 \mathrm{ab}$ & $16,2 \mathrm{a}$ & $21,7 \mathrm{abc}$ & $12,8 \mathrm{a}$ & $12,0 \mathrm{a}$ & $14,8 \mathrm{ab}$ \\
AABrGxGPY & $9,0 \mathrm{a}$ & $16,0 \mathrm{a}$ & $21,7 \mathrm{abc}$ & $12,0 \mathrm{a}$ & $12,0 \mathrm{a}$ & $14,8 \mathrm{ab}$ \\
AVBrGxGBrPF & $9,0 \mathrm{a}$ & $15,2 \mathrm{ab}$ & $20,7 \mathrm{bcd}$ & $12,0 \mathrm{a}$ & $12,0 \mathrm{a}$ & $14,0 \mathrm{ab}$ \\
AVBrGxGOA & $9,0 \mathrm{a}$ & $15,2 \mathrm{ab}$ & $20,7 \mathrm{bcd}$ & $12,0 \mathrm{a}$ & $12,0 \mathrm{ab}$ & $14,0 \mathrm{ab}$ \\
AVBrGxGPY & $9,2 \mathrm{a}$ & $15,7 \mathrm{a}$ & $21,5 \mathrm{abc}$ & $12,0 \mathrm{a}$ & $12,0 \mathrm{a}$ & $14,8 \mathrm{ab}$ \\
AVBrGxGRL & $8,5 \mathrm{ab}$ & $15,0 \mathrm{ab}$ & $21,0 \mathrm{abcd}$ & $12,0 \mathrm{a}$ & $12,0 \mathrm{a}$ & $16,0 \mathrm{a}$ \\
AVeBrJxGBrPF & $8,2 \mathrm{abc}$ & $15,0 \mathrm{ab}$ & $21,7 \mathrm{abc}$ & $\mathbf{1 2 , 4}$ & $\mathbf{1 2 , 0}$ & $\mathbf{1 4 , 4}$ \\
\hline Média & $\mathbf{7 , 6}$ & $\mathbf{1 4 , 8}$ & $\mathbf{2 1 , 1}$ & & & \\
\hline
\end{tabular}

Médias seguidas mesma letra na coluna não diferem entre si, pelo teste de Tukey, a $5 \%$ de probabilidade.

TABELA 3 - Dados médios da circunferência do coleto e em cultivares de coqueiro, no período de 1997 a 2000, em Neópolis- SE.

\begin{tabular}{lccc}
\hline \multicolumn{1}{c}{ Cultivar } & & CC & Ano 3 \\
\cline { 2 - 4 } & Ano 1 & Ano & $110,7 \mathrm{~cd}$ \\
\hline AABrG & $30,2 \mathrm{ab}$ & $91,1 \mathrm{~d}$ & $104,5 \mathrm{de}$ \\
AAM & $29,0 \mathrm{ab}$ & $88,4 \mathrm{~d}$ & $96,4 \mathrm{e}$ \\
AVC & $24,0 \mathrm{c}$ & $73,5 \mathrm{e}$ & $119,4 \mathrm{~cd}$ \\
AVBrG & $30,7 \mathrm{ab}$ & $92,2 \mathrm{~cd}$ & $118,6 \mathrm{~cd}$ \\
AVM & $27,5 \mathrm{ab}$ & $88,5 \mathrm{~d}$ & $108,6 \mathrm{cde}$ \\
AVeBrJ & $30,3 \mathrm{ab}$ & $90,4 \mathrm{~d}$ & $153,2 \mathrm{a}$ \\
AABrGxGBrPF & $39,8 \mathrm{a}$ & $115,4 \mathrm{ab}$ & $136,7 \mathrm{~b}$ \\
AABrGxGOA & $37,0 \mathrm{ab}$ & $106,2 \mathrm{bc}$ & $151,6 \mathrm{a}$ \\
AABrGxGPY & $38,4 \mathrm{ab}$ & $116,2 \mathrm{ab}$ & $156,9 \mathrm{a}$ \\
AVBrGxGBrPF & $39,7 \mathrm{a}$ & $125,5 \mathrm{a}$ & $153,3 \mathrm{a}$ \\
AVBrGxGOA & $39,6 \mathrm{a}$ & $114,2 \mathrm{ab}$ & $155,9 \mathrm{a}$ \\
AVBrGxGPY & $41,1 \mathrm{a}$ & $116,2 \mathrm{ab}$ & $158,0 \mathrm{a}$ \\
AVBrGxGRL & $41,2 \mathrm{a}$ & $117,8 \mathrm{ab}$ & $156,6 \mathrm{a}$ \\
AVeBrJxGBrPF & $39,2 \mathrm{a}$ & $113,9 \mathrm{ab}$ & $\mathbf{1 3 4 , 3}$ \\
\hline Média & $\mathbf{3 4 , 7}$ & $\mathbf{1 0 3 , 8}$ & \\
\hline
\end{tabular}

Médias seguidas mesma letra na coluna não diferem entre si, pelo teste de Tukey, a 5\% de probabilidade. 
TABELA 4 - Dados médios do comprimento da folha 3, do comprimento do limbo da folha 3 e comprimento do pecíolo da folha 3, em cultivares de coqueiro, no período de 1997 a 2000, em Neópolis-SE.

\begin{tabular}{|c|c|c|c|c|c|c|c|c|c|}
\hline \multirow[t]{2}{*}{ Cultivar } & \multicolumn{3}{|c|}{ CF3 } & \multicolumn{3}{|c|}{ CLF3 } & \multicolumn{3}{|c|}{ CPF3 } \\
\hline & Ano 1 & Ano 2 & Ano 3 & Ano 1 & Ano 2 & Ano 3 & Ano 1 & Ano 2 & Ano 3 \\
\hline AABrG & $1,1 \mathrm{~b}$ & $2,7 b c$ & $3,4 \mathrm{~b}$ & $0,8 \mathrm{bcd}$ & $2,3 \mathrm{~b}$ & $2,3 \mathrm{~cd}$ & $0,2 \mathrm{a}$ & $0,4 \mathrm{a}$ & $0,7 \mathrm{~d}$ \\
\hline AAM & $1,0 \mathrm{~b}$ & $2,6 b c$ & $3,4 b c$ & $0,8 \mathrm{~cd}$ & $2,2 b c$ & $2,3 \mathrm{~cd}$ & $0,2 \mathrm{a}$ & $0,4 \mathrm{a}$ & $0,7 \mathrm{~d}$ \\
\hline $\mathrm{AVC}$ & $0,9 \mathrm{~b}$ & $2,4 c$ & $3,0 \mathrm{c}$ & $0,6 \mathrm{~d}$ & $1,8 \mathrm{c}$ & $2,1 \mathrm{~d}$ & $0,2 \mathrm{a}$ & $0,4 \mathrm{a}$ & $0,9 \mathrm{~d}$ \\
\hline $\mathrm{AVBrG}$ & $1,1 \mathrm{~b}$ & $2,9 \mathrm{~b}$ & $3,6 b$ & $0,9 \mathrm{abcd}$ & $2,4 \mathrm{~b}$ & $2,6 \mathrm{~cd}$ & $0,3 \mathrm{a}$ & $0,5 \mathrm{a}$ & $0,9 \mathrm{~d}$ \\
\hline AVM & $1,0 \mathrm{~b}$ & $2,6 b c$ & $3,5 \mathrm{~b}$ & $0,8 \mathrm{~cd}$ & $2,2 b c$ & $2,5 \mathrm{~cd}$ & $0,4 \mathrm{a}$ & $0,4 \mathrm{a}$ & $1,0 \mathrm{~cd}$ \\
\hline $\mathrm{AVeBrJ}$ & $1,1 \mathrm{~b}$ & $2,8 b c$ & $3,4 \mathrm{~b}$ & $0,9 \mathrm{abcd}$ & $2,4 \mathrm{~b}$ & $2,3 \mathrm{~cd}$ & $0,3 \mathrm{a}$ & $0,3 \mathrm{a}$ & $1,0 \mathrm{bcd}$ \\
\hline $\mathrm{AABrGxGBrPF}$ & $1,7 \mathrm{a}$ & $3,7 \mathrm{a}$ & $4,3 \mathrm{a}$ & $1,1 \mathrm{abc}$ & $3,2 \mathrm{a}$ & $3,1 \mathrm{a}$ & $0,4 \mathrm{a}$ & $0,5 \mathrm{a}$ & $1,3 \mathrm{abc}$ \\
\hline AABrGxGOA & $1,6 \mathrm{a}$ & $3,5 \mathrm{a}$ & $4,1 \mathrm{a}$ & $1,1 \mathrm{abc}$ & $2,9 \mathrm{a}$ & $3,0 \mathrm{ab}$ & $0,4 \mathrm{a}$ & $0,6 \mathrm{a}$ & $1,2 a b c$ \\
\hline AABrGxGPY & $1,7 \mathrm{a}$ & $3,6 \mathrm{a}$ & $4,5 \mathrm{a}$ & $1,3 \mathrm{a}$ & $3,0 \mathrm{a}$ & $3,3 \mathrm{a}$ & $0,4 \mathrm{a}$ & $0,6 \mathrm{a}$ & $1,3 \mathrm{ab}$ \\
\hline AVBrGxGBrPF & $1,8 \mathrm{a}$ & $3,7 \mathrm{a}$ & $4,5 \mathrm{a}$ & $1,3 \mathrm{a}$ & $3,3 \mathrm{a}$ & $3,2 \mathrm{a}$ & $0,4 \mathrm{a}$ & $0,5 \mathrm{a}$ & $1,4 \mathrm{a}$ \\
\hline AVBrGxGOA & $1,8 \mathrm{a}$ & $3,7 \mathrm{a}$ & $4,5 \mathrm{a}$ & $1,2 \mathrm{ab}$ & $3,2 \mathrm{a}$ & $3,2 \mathrm{a}$ & $0,3 \mathrm{a}$ & $0,6 \mathrm{a}$ & $1,3 \mathrm{ab}$ \\
\hline AVBrGxGPY & $1,7 \mathrm{a}$ & $3,7 \mathrm{a}$ & $4,4 \mathrm{a}$ & $1,3 \mathrm{a}$ & $3,1 \mathrm{a}$ & $3,1 \mathrm{a}$ & $0,3 \mathrm{a}$ & $0,5 \mathrm{a}$ & $1,4 \mathrm{a}$ \\
\hline AVBrGxGRL & $1,8 \mathrm{a}$ & $3,6 \mathrm{a}$ & $4,4 \mathrm{a}$ & $1,3 \mathrm{a}$ & $3,2 \mathrm{a}$ & $3,1 \mathrm{a}$ & $0,4 \mathrm{a}$ & $0,5 \mathrm{a}$ & $1,4 \mathrm{a}$ \\
\hline AVeBrJxGBrPF & $1,7 \mathrm{a}$ & $3,6 \mathrm{a}$ & $4,2 \mathrm{a}$ & $1,2 \mathrm{ab}$ & $3,0 \mathrm{a}$ & $3,0 \mathrm{bc}$ & $0,3 \mathrm{a}$ & $0,5 \mathrm{a}$ & $1,3 \mathrm{ab}$ \\
\hline Média & 1,5 & 3,2 & 3,9 & 1,0 & 2,7 & 2,8 & 0,3 & 0,5 & 1,1 \\
\hline
\end{tabular}

Médias seguidas mesma letra na coluna não diferem entre si pelo teste de Tukey, a $5 \%$ de probabilidade.

TABELA 5 - Dados médios do número de folíolos da folha 3 e do comprimento dos folíolos da folha 3, em cultivares de coqueiro, no período de 1997 a 2000, em Neópolis-SE.

\begin{tabular}{l|l|l|l|l|c|c}
\hline Cultivar & \multicolumn{3}{|c|}{ NFoF3 } & \multicolumn{2}{c}{ CFoF3 } \\
\hline & Ano 1 & Ano 2 & Ano 3 & Ano 1 & Ano 2 & Ano 3 \\
\hline AABrG & $67,0 \mathrm{a}$ & $124,0 \mathrm{bcde}$ & $142,1 \mathrm{de}$ & $46,0 \mathrm{~cd}$ & $88,6 \mathrm{e}$ & $95,7 \mathrm{de}$ \\
\hline AAM & $64,2 \mathrm{a}$ & $117,5 \mathrm{de}$ & $145,3 \mathrm{de}$ & $43,7 \mathrm{~cd}$ & $81,8 \mathrm{e}$ & $93,7 \mathrm{e}$ \\
\hline AVC & $56,5 \mathrm{a}$ & $101,4 \mathrm{e}$ & $117,6 \mathrm{e}$ & $36,7 \mathrm{~d}$ & $66,7 \mathrm{e}$ & $77,8 \mathrm{e}$ \\
\hline AVBrG & $68,5 \mathrm{a}$ & $127,7 \mathrm{abcde}$ & $157,7 \mathrm{abcd}$ & $46,0 \mathrm{~cd}$ & $88,7 \mathrm{de}$ & $103,5 \mathrm{~cd}$ \\
\hline AVM & $59,7 \mathrm{a}$ & $119,1 \mathrm{cde}$ & $155,2 \mathrm{cde}$ & $44,2 \mathrm{~cd}$ & $85,4 \mathrm{e}$ & $107,4 \mathrm{bc}$ \\
\hline AVeBrJ & $63,2 \mathrm{a}$ & $123,4 \mathrm{bcde}$ & $136,7 \mathrm{e}$ & $44,2 \mathrm{~cd}$ & $85,4 \mathrm{e}$ & $107,4 \mathrm{bc}$ \\
\hline AABrGxGBrPF & $69,5 \mathrm{a}$ & $148,3 \mathrm{ab}$ & $183,7 \mathrm{ab}$ & $66,0 \mathrm{a}$ & $105,6 \mathrm{bcd}$ & $111,9 \mathrm{abc}$ \\
\hline AABrGxGOA & $68,7 \mathrm{a}$ & $132,8 \mathrm{abcde}$ & $150,6 \mathrm{de}$ & $59,7 \mathrm{ab}$ & $100,9 \mathrm{bcd}$ & $107,4 \mathrm{bc}$ \\
\hline AABrGxGPY & $69,7 \mathrm{a}$ & $143,1 \mathrm{abcde}$ & $167,9 \mathrm{abcd}$ & $62,0 \mathrm{a}$ & $102,5 \mathrm{bcd}$ & $110,0 \mathrm{abc}$ \\
\hline AVBrGxGBrPF & $73,0 \mathrm{a}$ & $152,5 \mathrm{a}$ & $185,7 \mathrm{a}$ & $66,0 \mathrm{a}$ & $105,8 \mathrm{bcd}$ & $115,2 \mathrm{ab}$ \\
\hline AVBrGxGOA & $72,2 \mathrm{a}$ & $149,8 \mathrm{ab}$ & $183,2 \mathrm{ab}$ & $65,5 \mathrm{a}$ & $111,8 \mathrm{a}$ & $117,7 \mathrm{a}$ \\
\hline AVBrGxGPY & $73,2 \mathrm{a}$ & $145,4 \mathrm{abc}$ & $181,2 \mathrm{abc}$ & $67,5 \mathrm{a}$ & $110,3 \mathrm{ab}$ & $119,4 \mathrm{a}$ \\
\hline AVBrGxGRL & $72,7 \mathrm{a}$ & $149,2 \mathrm{ab}$ & $184,6 \mathrm{ab}$ & $66,7 \mathrm{a}$ & $107,9 \mathrm{bcd}$ & $117,9 \mathrm{a}$ \\
\hline AVe BrJxGBrPF & $71,0 \mathrm{a}$ & $148,1 \mathrm{ab}$ & $180,4 \mathrm{abc}$ & $65,7 \mathrm{a}$ & $104,4 \mathrm{abcd}$ & $117,5 \mathrm{a}$ \\
\hline Média & $\mathbf{6 7 , 8}$ & $\mathbf{1 3 4 , 4}$ & $\mathbf{1 6 2 , 3}$ & $\mathbf{5 6 , 1}$ & $\mathbf{9 7 , 0}$ & $\mathbf{1 0 7 , 1}$ \\
\hline Med & & & & &
\end{tabular}

Médias seguidas mesma letra na coluna não diferem entre si, pelo teste de Tukey, a $5 \%$ de probabilidade.

\section{CONCLUSÃO}

1- As cultivares de coqueiro apresentam um comportamento diferente em relação ao ano para o desenvolvimento foliar, circunferência do coleto, comprimento da folha 3, comprimento do limbo da folha 3, comprimento do pecíolo da folha 3 , número de folíolos da folha 3 e comprimento dos folíolos da folha 3 .

2- A partir do primeiro ano após o plantio até o terceiro ano, o aumento no número de folhas vivas é constante, independentemente das cultivares.
3- O número de folhas emitidas entre as cultivares de coqueiro varia de 12 a 16 folhas no terceiro ano.

4- O maior aumento da circunferência do coleto, do comprimento de folha 3 , do comprimento do limbo da folha 3 , do número de folíolos da folha 3 e do comprimento do folíolo da folha 3 , entre as cultivares, ocorre no segundo ano em relação ao primeiro e terceiro anos, decrescendo a partir daí.

5-Os híbridos apresentaram maior desenvolvimento foliar avaliado, enquanto os anões, os menores, sendo o AVC o menor entre os anões. 


\section{REFERÊNCIAS}

ARAGÃO, W. M., COSTA, A. S. da, SANTOS, H. C. A. C., PEDROSO, G. T. Florescimento, produção e composição morfológica de frutos de cultivares de coqueiro. Agrotrópica, Bahia, v. 14, n. 3, p. 151-158, 2002.

CAMBOIM, L. A cultura do coqueiro. 2003 Disponível em: $<$ www.geocities.com/coqueiroanao/>. Acesso em: 25 jan. 2005.

CHILD, R. Coconuts. $2^{\text {nd }}$ ed. London: Longman, 1974.335 p. CRUZ, C. D.; REGAZZI, A. J. Modelos biométricos aplicados ao melhoramento genético. Viçosa: UFV, 1994.390 p.

FALCONER, D. S. Introdução á genética quantitativa. 2.ed. Viçosa: UFV, 1987.279 p.

FERRI, M. G. Botânica: morfologia externa das plantas (organografia). São Paulo: Melhoramentos, 1973.149 p.

FREMOND, Y.; ZILLER, R.; NUCÉ de LAMOTHE, M. El cocotero: técnicas agrícolas y producciones tropicales. Barcelona: Editorial Blume, $1975.236 \mathrm{p}$.

GOMES, F. P. Curso de estatística experimental. 11. ed. Piracicaba: Nobel, 1985. p. 24-26

MENON, K. P. V.; PANDALAI, K. M. The coconut palm: a monograph. Ernakulam: Indian Central Coconut Commitee, 1958. $384 \mathrm{p}$.
PASSOS, E. E. M.; SILVA, J. V. Determinação do estado hídrico do coqueiro através do método dendrométrico. Oléagineux, Paris. v. 46, n. 6, p. 233-238, 1991.

PASSOS, E.E.M. In: FERREIRA,J.M.S.; WARWICK,D.R.N.; SIQUEIRA, L.A. A cultura do coqueiro no Brasil. 2.ed. Brasília: EMBRAPA-SPI; Aracaju: EMBRAPA-CPATC, 1998. p. 57-72. PURSEGLOVE, J. W. Tropical crops monocotyldons. London: Longman, 1972.607p.

RAMOS, V. H. V. ; PINTO, A. C. Q. ; ARAGÃO, W. M. ; GOMES, A. C. ; JUNQUEIRA, N. T. V. ; ANDRADE, S. R. M. ; CORDEIRO, M. C. R. ; DIAS, J. N. ; ANDRADE, G. A. Comportamento de cultivares de coqueiro anão e híbrido no distrito federal. Revista Brasileira de Fruticultura, Jaboticabal, v. 26, n. 2, p. 363-365, 2004.

SOBRAL, L. F. Nutrição e adubação do coqueiro. In: FERREIRA, J.M.S.; WARWICK, D.R.N.; SIQUEIRA, L.A. (Org.). A cultura do coqueiro no Brasil. 2.ed. Brasília: EMBRAPA-SPI; Aracaju: EMBRAPA-CPATC, 1998. p. 129-157.

VENCOVSKY, R; BARRIGA, J. T. Conservação in vitro de recursos genéticos de plantas. In: TORRES, A.C.; CALDAS, L.S.; BUS, J.A. (Ed.) Cultura de tecidos e transformação genética de plantas. Brasília: Embrapa Comunicação para Transferência de Tecnologia, 1998. p. 297-330.

WORLD BANK. Coconut production: present status and priorities for research. Washington, DC: World Bank Technical Paper, $1991.150 \mathrm{p}$. 